\title{
A Comparative Analysis on the Curative Effects of Different Treatment for Infections Around Dental Implants
}

\author{
Hui Wang, Ting Tang, Min Jin, Lili Fang, Aihua Ou* \\ Hefei School of Stomatology, Anhui Medical University, Hefei Stomatological Hospital, Hefei 230001, Anhui Province,
} China

*Corresponding author: Aihua Ou, wanghui20121003@sina.com

\begin{abstract}
Objective: To analyze and evaluate the clinical efficacy of different treatment options for patients with peri-implant infection. Methods: The study period was from June 2019 to June 2020. Seventy-six patients with peri-implant infection that were treated in Stomatological Hospital of Hefei were selected as study samples. The patients were divided into a research group and a control group by random number table method in which the sample size of each group was $n=38$. Patients in the control group were treated with drugs combined with ultrasonic cleaning while patients in the research group were treated with guided bone regeneration combined with flap curettage. The indicators of the two groups were compared and analyzed. Results: The plaque index (PLI), probing depth (PD), and sulcus bleeding index (SBI) of the two groups after treatment were lower than those before treatment $(\mathrm{P}<0.05)$. There was no significant difference between the groups $(\mathrm{P}>0.05)$. However, the height of the implant marginal bone of the study group after treatment was higher than that of the control group $(\mathrm{P}<0.05)$. Conclusion: The use of guided bone regeneration combined with flap curettage for patients with infection around dental implants promotes the fusion of bone and implants. The clinical effect was significant and this should be comprehensively promoted in medical institutions at all levels.
\end{abstract}

Keywords: Dental implant; Infection; Treatment

Publication date: July 2021; Online publication: July 31, 2021

\section{Introduction}

Dental implants are the main clinical treatment for tooth loss. In the treatment, artificial materials are implanted as tooth roots by specific procedures and bone tissue retention support is obtained. After restoration treatment, the structure and function of the natural tooth can be approximated. ${ }^{[1]}$ Infection around dental implant is a major complication of clinical dental implant treatment which may lead to the loss of bone around the implant and also induce the loosening or loss of the implant which affects longterm implant restoration. For this reason, effective treatment interventions are needed. ${ }^{[2]}$ This study summarized and evaluated the basic clinical data of patients in Hefei Stomatological Hospital as well as analyzed and comprehensively studied the clinical efficacy of different treatment options for infection around dental implants.

\section{Materials and methods}

\subsection{General information}

The study period was from June 2019 to June 2020. Seventy-six patients with infection around dental implants at Hefei Stomatological Hospital were selected as study samples and were divided equally using 
the random number table model. The sample size in both the research and control group was $n=38$. The clinical data of the two groups of patients were summarized and comprehensively analyzed. The research group comprised of 22 male patients and 16 female patients, age ranging from 25 to 57 years old with an average age of $41.58 \pm 2.69$ years old. In the control group, there were 20 male patients and 18 female patients, age ranging from 27 to 55 years old with an average age of $41.32 \pm 2.75$ years old. The differences in the baseline clinical data had no effect on the conclusion of this study $(\mathrm{P}>0.05)$.

The inclusion criteria were inflammation of the gingival tissue or mucosal tissue around the implant, SBI greater than 1, PD of 3-5mm, mild or moderate resorption of the alveolar crest, and bone resorption of less than $4 \mathrm{~mm}$. The exclusion criteria were patients with multiple systemic diseases or patients who would not cooperate with the study.

\subsection{Method}

The patients in the control group were treated with drugs combined with ultrasonic cleaning. For each patient, the surface of the implant was treated with an ultrasonic cleaning device which effectively cleaned the tartar and pigment of the whole mouth. After meeting the relevant treatment intervention standards, the carbon fiber curette in Hefei Stomatology Hospital was used to perform subgingival cleaning. After the completion of the treatment, the periodontal and gingival pocket were rinsed with normal saline. Then, the soft tissues around the implant were carefully examined to determine if any bleeding occurred. If the bleeding were minimal, an appropriate amount of minocycline hydrochloride ointment would be injected at the bottom of the patient's gum pocket to completely surround the tooth. After the surgery, the patient was not allowed to suck, eat, or gargle for 1 hour, and the implant was treated daily with medicine and irrigation for a total of 4 treatments.

Each patient in the research group underwent guided bone regeneration combined with flap curettage, flap treatment underneath the periosteum, complete scraping of inflammatory granulation tissue on the surface of the implant, and abrasion of abnormal bone tips and ridges. Normal saline was used to repeatedly wash the surgical area, the bone defect area was filled with Bio-Oss bone powder, the surface defect area was measured, the appropriate size of Bio-Oss collagen was selected to accurately cover the periosteum, and the normal shape of the alveolar was restored after stitching the incision part.

\subsection{Evaluation}

The PLI (plaque index), PD (probing depth), and SBI (sulcus bleeding index) before treatment and 6 months after treatment were compared between the two groups. PD involved the use of a periodontal probe $(0.5 \mathrm{~mm})$ to measure the distance between the bottom of the implant pocket and the gingival margin, as well as the values of the tongue, lip, distal, and mesial surfaces respectively while taking the average value. PLI 0 was classified as sterile plaque, 1 meant that it was easy to produce plaque, 2 indicated visible plaque, and 3 indicated a large amount of plaque. SBI involved the use of a scaled periodontal probe to measure by moving along the $1 \mathrm{~mm}$ area below the gingival margin of the implant to evaluate the conditions of the sulci. Grade 0 indicated normal, grade 1 indicated mild edema, grade 2 indicated mild bleeding, grade 3 indicated massive bleeding that continued to expand at the gingival margin, and grade 4 indicated heavy bleeding that overflowed into the gingival margin.

The height of the implant marginal bone was measured before and 6 months after the treatment of the two groups of patients. X-rays were used to determine the mesial and mid-distal bone height in which the top area of the implant was used as a reference point. 


\subsection{Statistical analysis}

Statistical Package for the Social Sciences (SPSS) version 23.0 software was used to analyze various data. In this study, the measurement data were $(\bar{x} \pm \mathrm{s})$, the count data were $(\%)$, and the test methods were $\mathrm{t}$-test and chi-square $\left(\mathrm{X}^{2}\right) . \mathrm{P}<0.05$ indicated a difference between the groups.

\section{Results}

\subsection{Comparison of PLI, PD, and SBI before and after the treatment of the two groups}

The PLI, PD, and SBI of the two groups after treatment were lower than those before treatment $(\mathrm{P}<0.05)$ and there was no significant difference between the groups $(\mathrm{P}>0.05)$.

Table 1. Comparison of PLI, PD, and SBI before and after treatment between the two groups $(\bar{x} \pm s)$

\begin{tabular}{ccccccc}
\hline \multirow{2}{*}{ Group } & \multicolumn{2}{c}{ PLI (Minute) } & \multicolumn{2}{c}{ PD $(\mathbf{m m})$} & \multicolumn{2}{c}{ SBI (level) } \\
\cline { 2 - 6 } & $\begin{array}{c}\text { Prior } \\
\text { treatment }\end{array}$ & $\begin{array}{c}\text { After } \\
\text { treatment }\end{array}$ & $\begin{array}{c}\text { Prior } \\
\text { treatment }\end{array}$ & $\begin{array}{c}\text { After } \\
\text { treatment }\end{array}$ & $\begin{array}{c}\text { Prior } \\
\text { treatment }\end{array}$ & $\begin{array}{c}\text { After } \\
\text { treatment }\end{array}$ \\
\hline $\begin{array}{c}\text { Control group } \\
(\mathrm{n}=38)\end{array}$ & $2.89 \pm 0.35$ & $1.12 \pm 0.28^{*}$ & $4.52 \pm 0.66$ & $2.15 \pm 0.33^{*}$ & $3.59 \pm 0.68$ & $1.22 \pm 0.43^{*}$ \\
$\begin{array}{c}\text { Research } \\
\text { group (n=38) }\end{array}$ & $2.75 \pm 0.42$ & $1.15 \pm 0.35^{*}$ & $4.58 \pm 0.61$ & $2.17 \pm 0.36^{*}$ & $3.54 \pm 0.71$ & $1.27 \pm 0.45^{*}$ \\
t value & 1.578 & 0.412 & 0.411 & 0.252 & 0.313 & 0.495 \\
P value & 0.118 & 0.681 & 0.681 & 0.801 & 0.754 & 0.621 \\
\hline
\end{tabular}

Note: Comparing with before treatment, the difference was significant, $* \mathrm{P}<0.05$

\subsection{Comparison of the marginal bone height before and after treatment between the two groups}

After treatment, the implant marginal bone height of the research group was higher than that of the control group $(\mathrm{P}<0.05)$.

Table 2. Comparison of the marginal bone height before and after treatment between the two groups $(\bar{x} \pm \mathrm{s}$, $\mathrm{mm})$

\begin{tabular}{ccc}
\hline Group & Prior treatment & After treatment \\
\hline Control group $(\mathrm{n}=38)$ & $-3.85 \pm 1.27$ & $-3.27 \pm 1.03$ \\
Research group $(\mathrm{n}=38)$ & $-3.79 \pm 1.15$ & $-2.19 \pm 0.84$ \\
t value & 0.215 & 5.009 \\
P value & 0.829 & 0.000 \\
\hline
\end{tabular}

\section{Discussion}

Infection around dental implants is one of the multiple complications of clinical dental implants. The main cause is related to poor oral hygiene leading to pathogens invading the human body. The clinical manifestations are similar to symptoms related to periodontitis. Infection around dental implants can cause gum swelling, bleeding, and the formation of periodontal pockets. Failure to effectively treat this may even cause implants to loosen or fall off. ${ }^{[3]}$

The treatment regime for infection around dental implants mainly includes drug intervention, manual curettage, cleaning, and surgical treatment. Drugs combined with ultrasonic cleaning is a routine clinical 
treatment of the disease. Its main advantage is that it has a broad-spectrum antibacterial property, reduces the relatively large number of drug-resistant bacteria, and promotes periodontal tissue regeneration. ${ }^{[4]}$ After the ultrasonic cleaning is completed, sandblasting, polishing, and surface repair are often used to improve the smoothness and cleanliness of the surface. Conventional drugs including chlorhexidine, tetracycline, minocycline hydrochloride, etc. can be combined with ultrasonic cleaning to provide significant therapeutic effects. ${ }^{[5]}$

Guided bone regeneration combined with flap curettage is a new clinical treatment for infection around dental implants. During the treatment, guided bone regeneration is effectively integrated with cleaning. Periodontal ligament materials are used as the treatment barrier. With thorough cleaning, the cause can be removed. Germs can also be prevented during root growth in addition to the healing of the gum epithelium and the contact between the root area and the gingival connective tissue would be blocked. It can also form a specific spatial structure and guide the periodontal ligament cells with strong attachment ability to occupy the root. It induces the formation of cementum in the root area of the periodontal pocket, accelerates the regeneration of periodontal tissue, and produces healing abutment. ${ }^{[6]}$ Guided bone regeneration combined with flap curettage can completely remove pathogenic bacteria that induce infection around dental implants by injecting bone powder, cleaning, and covering with collagen membranes which can also accelerate the fusion of bone and implants, significantly increase the height of the implant margins, and eventually achieve the desired therapeutic effect. ${ }^{[7]}$ If the patient has significant bone resorption around the implanted denture and the depth of the periodontal pocket in the area surrounding the implant is more than $5 \mathrm{~mm}$ in combination with bone damage, it is recommended to prioritize guided bone regeneration combined with flap curettage treatment in order to improve the fixation effect of the implant and effectively control the infection.

By summarizing and comprehensively analyzing the data of the two groups, the PLI, PD, and SBI of the two groups after treatment were better than those before treatment, and there was no significant difference between the two groups. This suggests that drugs combined with ultrasound treatment as well as guided bone regeneration combined with flap curettage are effective treatment measures for infection around dental implants. After treatment, plaque can be effectively removed and clinical symptoms such as bleeding can be relieved. The height of the marginal bone in the research group after treatment was higher than that in the control group, suggesting that guided bone regeneration combined with flap curettage can promote the effective fusion of bone and implant and its comprehensive clinical efficacy is better than that of drugs combined with ultrasonic cleaning. However, there are certain differences in the condition of patients in regard to their ability to absorb drugs and the infection around different dental implants. There are also large differences in the clinical effects of the above treatments. Therefore, it is necessary to evaluate the infection and periodontal status of the patients in order to come up with a reasonable treatment plan. In addition to the above treatment options, ultrasonic scalpel subgingival scaling combined with subgingival sandblasting and drugs is a new clinical intervention for infection around dental implants. In this treatment, ultrasonic vibration can effectively remove calculus and plaque in which they can be destroyed by acoustic effects. In regard to the internal structure of bacteria, the sandblasting treatment combined with glycine can provide a therapeutic effect. In addition to the conventional minocycline hydrochloride in the selection of therapeutic drugs, broad-spectrum antibacterial drugs such as Palio can also be selected to improve the antiinfective effect. ${ }^{[8]}$

Based on the above analysis and research, it can be seen that guided bone regeneration combined with flap curettage for patients with infection around dental implants can promote the fusion of bone and implants. The clinical effect was significant and should be comprehensively promoted in medical institutions at all levels. However, the sample size of this study was small, there was a lack of comparative analysis and research of the same type of data, and the duration of the research was short in which the 
process design and other aspects required further improvement. The effective treatment of infection around dental implants still requires continuous evaluation and analysis.

\section{Project}

This article belongs to a project titled "Effect of Systematic Health Education on Prevention of Peri-implant Diseases".

\section{Disclosure statement}

The author declares no conflict of interest.

\section{References}

[1] Zou Z, Dang H, Sun G, 2019, Comparison of the Effects of Ultrasound Cleaning Combined with Drugs and Flap Curettage Combined with Guided Bone Regeneration in the Treatment of Infection Around Dental Implants. China Practical Medical Journal, 46(17): 82-4.

[2] Wang X, Liu S, Wu X, 2018, Clinical Study of Flap and Curettage Combined with Guided Bone Regeneration in the Treatment of Infection Around Dental Implants. Modern Journal of Integrated Traditional Chinese and Western Medicine, 27(3): 279-81.

[3] Cao Y, Xie H, Han X, et al., 2020, The Effect of Different Retention Methods of Implant Dentures on the Tissue Surrounding the Implants in Patients. Clinical Misdiagnosis and Mistreatment, 33(2): 99103.

[4] Liu Z, Wu L, 2019, Analysis of the Distribution of Pathogenic Bacteria and Inflammation Index Levels Around Oral Implants in the Elderly. Chinese Primary Medicine, 26(11): 1381-3.

[5] Chen X, He T, Yang F, et al., 2019, Analysis of the Clinical Effect of Minocycline in the Treatment of Peri-Implant Infections in Diabetic Patients. Chinese Journal of Hospital Infection, 29(5): 741-4.

[6] Sun F, Zhou Y, Yan J, et al., 2019, Study on the Changes of Inflammatory Factors in Gingival Crevicular Fluid, Streptococcus sanguis and Porphyromonas gingivalis in Patients with PeriImplantitis. Chinese Journal of Hospital Infection, 29(1): 120-3.

[7] Sun X, Lv B, 2020, The Effect of Immediate Restoration and Delayed Restoration of a Single Tooth Immediately After Implantation in the Aesthetic Area on the Formation of Surrounding Gingival Soft Tissue. Journal of Baotou Medical College, 36(8): 47-50.

[8] Xue C, Tan Y, Wang Y, 2020, Correlation Analysis of Oral Helicobacter Pylori Infection and PeriImplant Inflammation and its Clinical Indicators. Journal of Huazhong University of Science and Technology (Medical Edition), 49(6):728-31. 